\title{
VibraTilt: Accelerometer \& Gyroscope measurement app
}

\author{
Khairani Muhammad Ng${ }^{1}$, Phi-Vu Nguyen ${ }^{1}$ and Samuel Ken-En Gan ${ }^{1,2^{*}}$
}

\begin{abstract}
Smartphones doubling up as laboratory equipment are increasingly in demand for convenience and portability. VibraTilt is a free smartphone app with a simple interface on both the iOS and Android operating systems that functions as both an accelerometer and a gyroscope. Leveraging on in-built sensors, the portable accelerometer bubble level can be used in labs to determine the tilt of surfaces. The gyroscope measures the rotation in threedirections-axes and their magnitude of change for calibrating equipment, measuring vibrations, stability of laboratory equipment, and measuring tremors clinically. For setting thresholds and visualization, a graph showing the change in the $x, y$ and $z$ values is incorporated in the Android version. Together with the customizable feature to record the number of shakes within a certain time frame, VibraTilt does more than unlock the smartphone to displace two equipment in the laboratory, but can be used in a variety of purposes.
\end{abstract}

\section{Introduction}

Reproducibility is an important criteria of experimental research. As such, calibrated equipment and standardised measures are reported in experimental research articles. To ensure optimal conditions, additional devices for calibration or comparisons are extremely welcomed. With the widespread use of smartphones and mobile devices with multiple sensors, there is great potential for phones to be leveraged as research equipment themselves. They can function as the desired "second opinion" devices, and all that is required to unlock these potential are mobile apps (http://www.biospectrumasia.com/biospectrum/ opinion/220592/use-smartphone-apps-biomedical-research). Mobile apps are now beginning to make an impact on research processes ranging from displacing equipment e.g.GelApp (Sim et al. 2015). Although a wide range of science-related apps have been created for aiding research, more remains to be done.

Both the gyroscope and accelerometer are widely used in scientific disciplines and daily life e.g. sports. Gyroscopes can be used to monitor earthquakes, precision engineering processes, neurological tremors such as Parkinson's disease, or the stability of a process and ride

\footnotetext{
* Correspondence: samuelg@bii.a-star.edu.sg

${ }^{1}$ Bioinformatics Institute, Agency for Science, Technology, and Research (A*STAR), Singapore 138671, Singapore

${ }^{2}$ p53 Laboratory, Agency for Science, Technology, and Research (A*STAR), Singapore 138648, Singapore
}

(e.g. in protein crystallization processes and cell fusion procedures). Accelerometers measure the tilt and levelledness of surfaces that are important for the construction industry and sensitive cell seeding experiments or tiltsensitive experiments in experimental laboratories. While they are useful, devices with such capabilities are not commonly available. Therefore, to address the need to make such devices more common for incorporation into the above examples, VibraTilt was developed as a free Android and iOS app to unlock the popular smartphone to have these additional functions.

Although there are numerous apps that unlock these smartphone sensors, the distinguishing feature of VibraTilt is the simple interface and the customizable threshold, sensitivity, and time limit for "number of shakes". This allows VibraTilt to function at another level of use ranging from sports, clinical, precision engineering, calibration, etc.

\section{Development}

VibraTilt for Android was developed using version 4.4 of the Eclispe Integrated Development Environment, Luna (https://eclipse.org/), as well as open source code from aChartEngine (http://www.achartengine.org/) for the generation of logarithmic graphs.

VibraTilt App for iOS was developed using version 6.3.2 of the Xcode Integrated Development Environment, utilising open source libraries: RGB colour sliders (https:// 

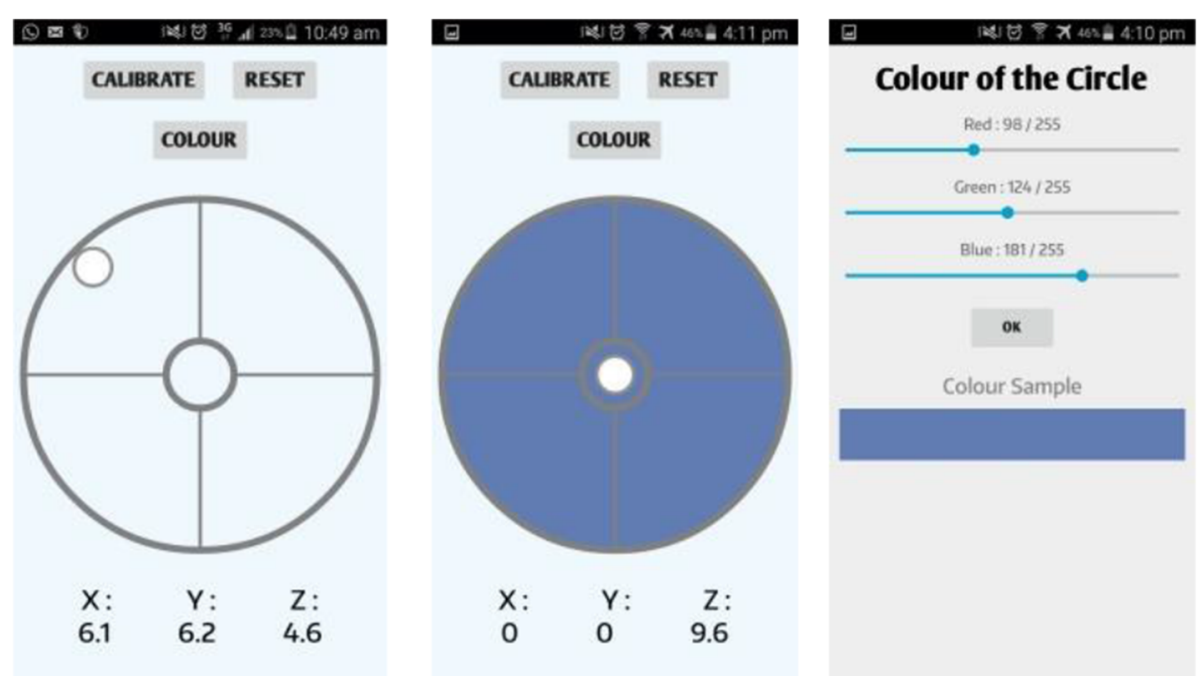

Fig. 1 Accelerometer Function. The accelerometer animation shows an animated bubble floating in a round half-sphere (left). When the phone/device is levelled, the bubble would be in the centre (centre). For better visualization, the colour of the liquid in the half-sphere can be changed (right)

github.com/eappel/RGBColorSlider) for changing the colour of the accelerometer bubble.

\section{Accelerometer function}

VibraTilt measures the tilt of an object surface in the accelerometer function. Tilting the phone moves the animated bubble from the centre circle as seen in real weighing balances in laboratories. The app detects three types of tilt in the 3 axes of $x, y$, and $z$ with values to a decimal point (Fig. 1). Should the smartphone require re-calibration, pressing the "Calibrate" button sets the bubble to the centre and the axes values to "0". Pressing the "Reset" button restores the accelerometer back to factory defaults. For visualization purposes, the colour of
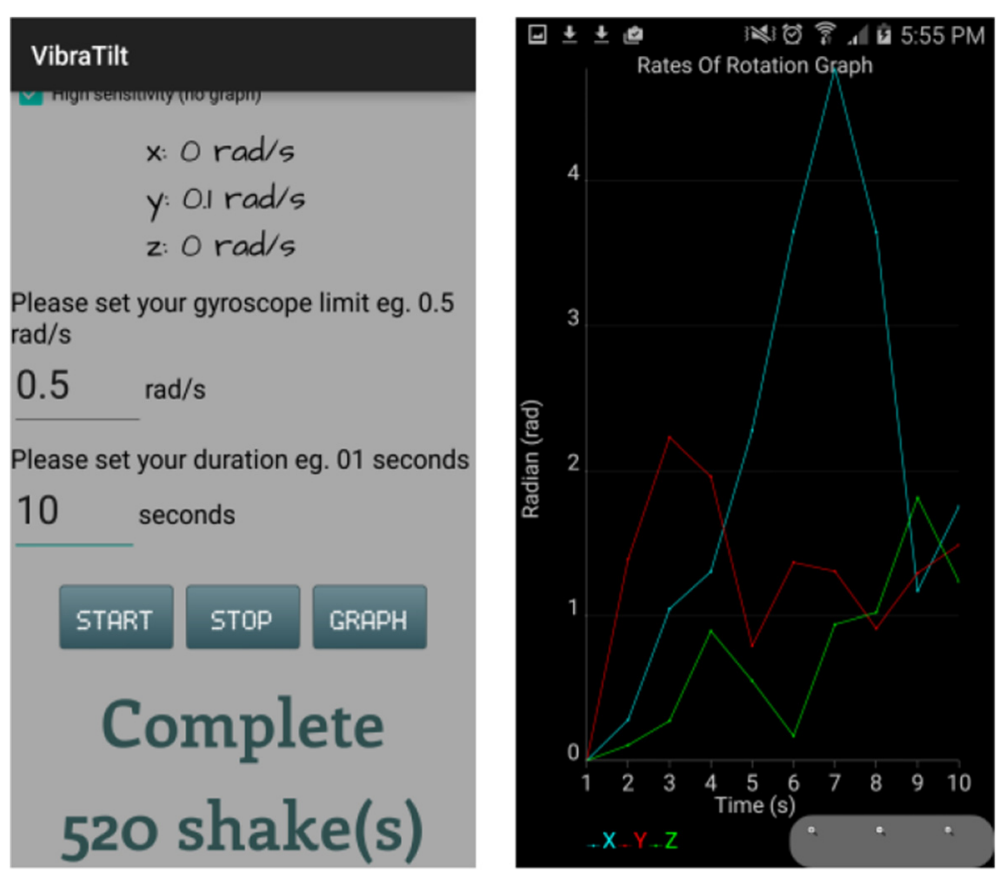

Fig. 2 Gyroscope Function. The gyroscope function measures the number of rotations past a set limit (left). For setting the limits, the graph (Android) can be used as a guide for specific activities (right) 
the circle can be changed with the "Colour" button which leads to a panel with three sliders corresponding to the RGB colour scheme (Fig. 1).

\section{Gyroscope function}

The gyroscope function (see Fig. 2) allows users to define the range of $x, y$, and $z$ axes (measured in $\mathrm{rad} / \mathrm{s}$ movements for measurement. The key unique feature of VibraTilt is the function to measure shakes in a set set time and threshold. For activity specific-parameters, users can set any number for a trial run and use the graph function (available only on Android and limited to measuring movements at every 10 milliseconds) to determine the desired magnitude of the movement of the target activity. Once the limits are set, pressing the "Start" button initiates the countdown of the set time limit and the number of rotations that exceed the defined $\mathrm{rad} / \mathrm{s}$. To end the measurement early, users can press the "Stop" button at any time. In the graph panel (only in Android), users can view the rate of rotation, where the radians of the axes are plotted against time (Fig. 2 bottom right). For measuring fast movements, a high sensitivity option is also available (movements are measured every millisecond). However, this feature disables the graph function due to the limitations of current smartphone processing capabilities for plotting high sensitivity data. For reports, screenshots can be taken at any point.

\section{Applications}

In the experimental laboratory, VibraTilt can be used to measure the stability and angle of surfaces for calibrating equipment and as a portable gyroscope for sensitive experiments. The key feature of measuring movement in a set time limit and magnitude allows the accelerometer function to do more than standardize shaking experiments, but also for neurological research involving tremors and even gait (LeMoyne et al. 2010). Outside the experimental laboratory, VibraTilt can be used for exercise and leisure purposes (pedometer, measuring mobile phone vibrations, or for determining if a piece of table/chair is tilted, etc).

\section{Conclusion}

VibraTilt functions as both a gyroscope and an accelerometer leveraging on the portability and convenience of smartphones/tablets. With added functions to calibrate, reset, and colour changes of the accelerometer together with the gyroscope measurement and graph function, much can be done with VibraTilt. In fact, VibraTilt marks the first such apps that is generic enough for both scientific research use, as well as for leisure.

\section{Availability and implementation}

VibraTilt (for Android and iOS) is freely available on the Google Play Store and Apple App Store as 'VibraTilt'. More details on the app can be found at www.facebook.com/ APDLab.

The 'VibraTilt' user guide is available at http:// tinyurl.com/VibraTilt and a video tutorial is also available on the Google Play Store.

The accuracy of the gyroscope and accelerometer is dependent on the smartphone sensors, therefore variations are expected when used on different devices.

\section{Competing interests \\ The authors declare no conflict of interest.}

\section{Authors' contributions}

KMN contributed to the manuscript, designed and made the app. PVN assisted in the graph and plotting aspect of the gyroscope. SKEG directed and conceived the idea of the app, as well as directed the writing of the application notes. All authors have read and approved the final version of the manuscript.

\section{Acknowledgements}

Funding: This work is funded by JCO1334i00050 grant, from the Joint Council Office, Agency for Science, Technology, and Research, Singapore. We thank Mr. Iman Fahman for helping with the accelerometer layout, Mr Sim Jia Zhi for technical assistance, and Mr. Dillon Lim for his help in this app note preparation.

Received: 23 November 2015 Accepted: 6 April 2016

Published online: 18 April 2016

\section{References}

LeMoyne R, Mastroianni T, Cozza M, Coroian C, Grundfest W. Implementation of an iPhone as a wireless accelerometer for quantifying gait characteristics. In Engineering in Medicine and Biology Society (EMBC), 2010 Annual International Conference of the IEEE. pp. 3847-3851. IEEE.

Sim JZ, Nguyen PV, Lee HK, Gan SKE. "GelApp: Mobile gel electrophoresis analyzer". Nature Methods Application Notes. April 2015. doi:10.1038/an9643. Use of Smartphone Apps for Biomedical Research - BioSpectrum Asia, 01-Jun-2015. [Online]. Available: http://www.biospectrumasia.com/biospectrum/opinion/ 220592/use-smartphone-apps-biomedical-research. Accessed 01 Jun 2015.

\section{Submit your manuscript to a SpringerOpen ${ }^{\circ}$ journal and benefit from:}

- Convenient online submission

- Rigorous peer review

- Immediate publication on acceptance

- Open access: articles freely available online

- High visibility within the field

- Retaining the copyright to your article

Submit your next manuscript at springeropen.com 